\title{
Gua Lou Gui Zhi decoction exerts neuroprotective effects on post-stroke spasticity via the modulation of glutamate levels and AMPA receptor expression
}

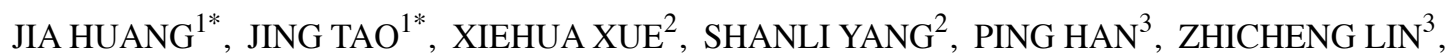 \\ WEI XU ${ }^{4}$, JIUMAO LIN ${ }^{5}$, JUN PENG ${ }^{5}$ and LIDIAN CHEN ${ }^{1}$ \\ ${ }^{1}$ College of Rehabilitation Medicine, ${ }^{2}$ MOE Key Laboratory of Traditional Chinese Medicine on Osteology and Traumatology \\ and Exercise Rehabilitation, ${ }^{3}$ Fujian Key Laboratory of Exercise Rehabilitation, ${ }^{4}$ College of Chinese Materia Medica, \\ ${ }^{5}$ Academy of Integrative Medicine, Fujian University of Traditional Chinese Medicine, \\ Minhou Shangjie, Fuzhou, Fujian 350108, P.R. China
}

Received November 13, 2012; Accepted January 11, 2013

DOI: 10.3892/ijmm.2013.1262

\begin{abstract}
Spasticity is one of the most physically debilitating disabilities following stroke and may slow down the potential success of rehabilitation. Glutamate and $\alpha$-amino-3-hydroxy5-methyl-4-isoxazolepropionic acid (AMPA) receptors have been shown to play a crucial role in spasticity following cerebral ischemia/reperfusion (I/R) injury. Gua Lou Gui Zhi decoction (GLGZD) is a well-known traditional Chinese formula that has long been used clinically in China to treat muscular spasticity following stroke, epilepsy or spinal cord injury. However, the precise mechanisms behind its neuroprotective and anti-spasticity effects remain poorly understood. In the present study, using a rat model of focal cerebral I/R injury, we evaluated the neuroprotective and anti-spasticity effects of GLGZD and investigated the underlying mechanisms. We found that GLGZD improved neurological deficits and reduced infarct volumes in cerebral I/R-injured rats. In addition, GLGZD reduced cerebral ischemic spasticity since it improved the screen test and Hoffman's reflex (H-reflex) scores. It also reduced glutamate levels in the cerebrospinal fluid and altered the expression of the AMPA receptor subunits. Our data demonstrate that GLGZD exerts neuroprotective and
\end{abstract}

Correspondence to: Dr Lidian Chen, College of Rehabilitation Medicine, Fujian University of Traditional Chinese Medicine, 1 Huatuo Road, Minhou Shangjie, Fuzhou, Fujian 350108, P.R. China E-mail: cld@fjtcm.edu.cn

*Contributed equally

Abbreviations: GLGZD, Gua Lou Gui Zhi decoction; MCAO, middle cerebral artery occlusion; CSF, cerebrospinal fluid; UMNS, upper motor neuron syndrome; AMPA, $\alpha$-amino-3-hydroxy-5-methyl4-isoxazolepropionic acid; TTC, 2,3,5-triphenyl tetrazolium chloride

Key words: spasticity, cerebral ischemia, glutamate, $\alpha$-amino-3hydroxy-5-methyl-4-isoxazolepropionic acid receptors, traditional Chinese medicine, Gua Lou Gui Zhi decoction anti-spasticity effects in a cerebral ischemia model via the modulation of glutamate levels and AMPA receptor expression.

\section{Introduction}

Stroke is a leading cause of serious and long-term disability in adults. One of the most physically debilitating disabilities is spasticity, defined as movement disorder (1). As one component of the upper motor neuron syndrome (UMNS) (2), spasticity is characterized by a velocity-dependent increase in tonic stretch reflexes (muscle tone) with exaggerated tendon jerks, resulting from hyperexcitablility of the stretch reflex. Spasticity one year after the event of stroke has been reported to occur in up to $38 \%$ of patients (3-5). Spasticity has a disabling effect on patients through reduced mobility and pain, which may slow down the potential success of rehabilitation (6), and eventually significantly affects the quality of life in stroke survivors.

Although the pathogenic mechanisms of spasticity are not yet well understood, glutamate-induced excitotoxicity is considered to be involved in this condition; thus, the suppression of excitation (glutamate) is considered as a means of treating spasticity $(7,8)$. Glutamate functions as a neurotransmitter in the majority of excitatory synaptic signals in the mammalian brain. It is the major excitatory neurotransmitter in the central nervous system (CNS), and is the agonist of two types of glutamate receptors, metabotropic and ionotropic glutamate receptors. $\alpha$-amino-3-hydroxy-5-methyl-4-isoxazolepropionic acid (AMPA) receptors are one of the ionotropic glutamate receptors for the fast excitatory synaptic transmission in the CNS. The binding of glutamate to post-synaptic AMPAtype glutamate receptors induces depolarizations leading to neuronal firing. AMPA receptor subunits (GluR1, GluR2, GluR3 and GluR4) play a crucial role in motor function following cerebral ischemia. It has been shown that AMPA subunits are upregulated in immunoreactive hypertrophic astrocytes in the CA1 hippocampal region after transient forebrain ischemia (9). Moreover, AMPA subunit expression has been shown to be significantly increased in reactive astrocytes during the chronic stages of ischemic spastic paraplegia (10), 
indicating that AMPA receptors are strongly associated with spasticity following cerebral ischemia.

A variety of therapeutic approaches are available for poststroke spasticity, including physical and occupational therapy, neurosurgery and orthopedic surgery, as well as oral medications. The mainstay of pharmacotherapy includes botulinum toxin, which functions by diminishing peripheral cholinergic activity at the neuromuscular junction, dantrolene sodium which inhibits the release of calcium from the sarcoplasmic reticulum, and a group of medications such as baclofen, diazepam and clonidine which act centrally (11-14). However, these medication procedures generally require repetition and may have troubling side-effects, such as permanent weakness, dysesthesias and causalgia (15).

Natural products, including traditional Chinese medicine (TCM), have relatively fewer side-effects as compared to modern chemotherapeutics and have been used for thousands of years as important alternative remedies for a variety of diseases. Gua Lou Gui Zhi decoction (GLGZD) is a wellknown traditional Chinese formula that was first recorded in 'Essentials from the Golden Cabinet' written during the Eastern Han Dynasty, around 210 AD. GLGZD consists of a combination of six herbs, including Trichosanthis Radix, Ramulus Cinnamomi, Paeonia lactiflora, Glycyrrhiza, Zingiber officinale Roscoe and Fructus Jujubae. GLGZD has long been used clinically in China to treat muscular spasticity following stroke, epilepsy or spinal cord injury (16-18). Our previous study demonstrated that GLGZD is effective in the treatment of post-stroke spasticity, improving the Fugl-Meyer score and Barthel Index score (unpublished data). In order to further elucidate the mode of action of GLGZD, in the present study, we used a focal cerebral ischemia/reperfusion (I/R) inujry rat model to evaluate the therapeutic efficacy of GLGZD against cerebral ischemia and spasticity, and investigated the underlying molecular mechanisms.

\section{Materials and methods}

Materials and reagents. GluR1, GluR2, GluR3 and GluR4 antibodies were provided by Abcam (Cambridge, MA, UK). $\beta$-actin antibodies and horseradish peroxidase (HRP)conjugated secondary antibodies were obtained from Cell Signaling Technology (Beverly, MA, USA). All other chemicals used, unless otherwise stated, were obtained from Sigma Chemical Co. (St. Louis, MO, USA).

Animals. Male Sprague-Dawley rats (with an initial body weight of $\sim 250 \mathrm{~g}$ ) were obtained from Shanghai SLAC Laboratory Animal Co., Ltd. (Shanghai, China) and housed in standard cages. A 12-h light/dark cycle was used throughout. Food and water were provided ad libitum during the experiment. All animal treatments were strictly in accordance with International Ethics Guidelines and the National Institutes of Health Guidelines Concerning the Care and Use of Laboratory Animals, and the experiments were approved by the Institutional Animal Care and Use Committee of Fujian University of Traditional Chinese Medicine, Fuzhou, China.

Establishment of cerebral ischemic spasticity and animal grouping. A cerebral ischemia model was established by middle cerebral artery occlusion (MCAO) as described previously (19). Briefly, after the rats were anesthetized with $10 \%$ chloral hydrate by intraperitoneal injection, the left common carotid artery (CCA), the left external carotid artery (ECA) and internal carotid artery (ICA) were carefully exposed by a midline neck incision. The left middle cerebral artery (MCA) was occluded by introducing an embolus through the ICA. Focal cerebral ischemia commenced when the tip of the catheter reached the origin of the MCA ( 18-22 mm). Mild resistance indicated that the embolus was properly lodged in the anterior cerebral artery, thus blocking blood flow to the MCA. Reperfusion was achieved by pulling out the thread after 120 min of occlusion to restore blood supply to the MCA area, and the left CCA and ECA were ligated. The rectal temperature of the rats was maintained at $37^{\circ} \mathrm{C}$ during the surgical procedures. After surgery the rats were allowed to recover in pre-warmed cages. Following the induction of ischemia, spasticity were examined by a screen test and Hoffman's reflex (H-reflex).

The rats were randomly divided into three groups $(n=8)$ as follows: i) sham-operated control (SC) group: rats underwent a neck dissection and coagulation of the ECA, but no occlusion of the MCA; ii) ischemia control (IC) group: the blood flow of the left MCA was blocked for $120 \mathrm{~min}$, followed by reperfusion; iii) GLGZD: the surgical procedure in the GLGZD group was the same as that in the IC group. Immediately after recovery from surgery, the rats received GLGZD at a concentration of $1.16 \mathrm{~g} / \mathrm{ml}$ daily for a period of seven days.

Preparation of herbal extracts. According to the original prescription from 'Essentials from the Golden Cabinet', the decoction comprised: Trichosanthis Radix, Ramulus Cinnamomi, Paeonia lactiflora, Glycyrrhiza, Zingiber officinale Roscoe and Fructus Jujubae at a ratio of 3:3:3:2:3:3. Dried crude drugs were purchased from Tongrentang Chinese Medicine Pharm (Fuzhou, China), a well-known and timehonored brand name in the TCM industry in China. They were identified and confirmed by the College of Pharmacology, Fujian University of Traditional Chinese Medicine. Ramulus Cinnamomi was pulverized to 100 mesh. The residual five dried materials were extracted twice with boiling water for $1.5 \mathrm{~h}$. The obtained solution was combined, filtrated (cotton gauze) and concentrated by using a rotary evaporator to a final concentration of $1.16 \mathrm{~g} / \mathrm{ml}$. The powder of Ramulus Cinnamomi was accurately weighed and added to the drug solution by stirring vigorously to produce a more uniform solution. The decoction was obtained for further use.

High-performance liquid chromatography (HPLC) fingerprint. An HPLC fingerprint was used to control the quality of the GLGZD extract in our study. A Sampark LC-20A series HPLC system (Sampark Technology, Japan) with a PDA detector and a Diamonsil C18 column (4.6x250 mm, $5 \mu \mathrm{m}$ particle size) was used for HPLC analysis. The UV spectra were recorded in the range $230-400 \mathrm{~nm}$, and the chromatographic peaks were measured at a wavelength of $230 \mathrm{~nm}$. The mobile phase consisted of solvent A (acetonitrile; Merck, USA) and solvent B ( $0.1 \%$ phosphoric acid/water, v/v). All agents were HPLC grade. The gradient procedure was used as follows: $5 \% \mathrm{~A}$ for $0 \mathrm{~min}, 32 \% \mathrm{~A}$ for $0-45 \mathrm{~min}, 48 \% \mathrm{~A}$ for $45-60 \mathrm{~min}$ and 


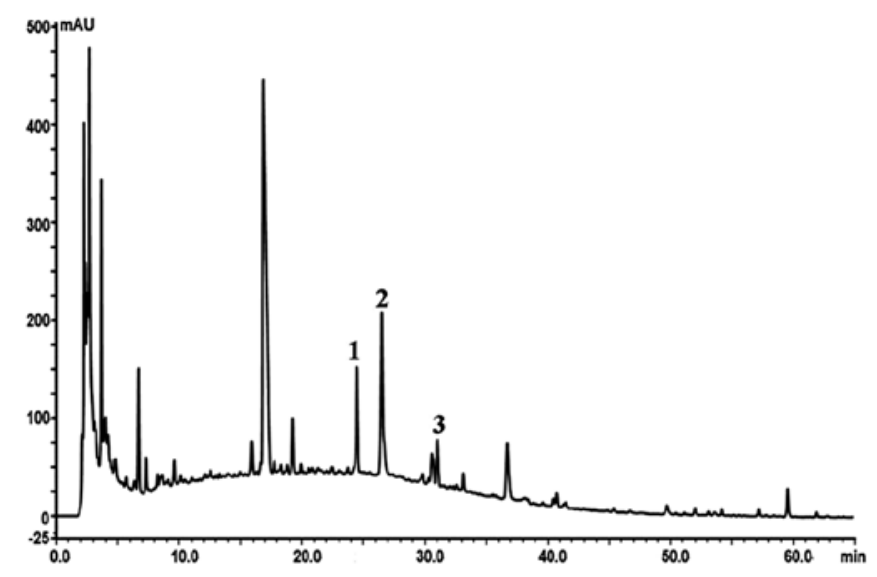

Figure 1. Chromatographic profile of Gua Lou Gui Zhi decoction (GLGZD) In order to control the quality of the GLGZD extract, the peak areas of R1, $\mathrm{R} 2$ and $\mathrm{R} 3$ should be in an optimum ratio of 15:21:7.

$48 \%$ A for $60-65 \mathrm{~min}$. The flow rate was $1.0 \mathrm{ml} / \mathrm{min}$ and the column temperature was set at $30^{\circ} \mathrm{C}$. Based on the fingerprint we established an optimum and easily controlled procedure for preparing the GLGZD extract as mentioned above (Fig. 1). If the peak areas of $\mathrm{R} 1, \mathrm{R} 2$ and $\mathrm{R} 3$ are in an optimum ratio of $15: 21: 7$, all results from the experiment would readily be reproducible.

Scoring of neurological deficits. At $2 \mathrm{~h}$ after cerebral ischemia and for the following seven days, the neurological deficit score was examined in a blinded fashion as described previously (19): score 0, no neurological deficit; score 1 (failure to extend the right forepaw fully), mild deficits; score 2 (circling to the right) and score 3 (falling to the right), moderate deficits; and score 4 (loss of walking), severe deficits. In brief, the rats with a score of 0 or 4 were excluded from the experiment.

Scoring of screen test. At $2 \mathrm{~h}$ after cerebral ischemia and for the following seven days, the screen test scores were examined in a blinded fashion as described previously (20). To measure the muscle tone, strength, stamina and balance, a net screen was used. The net screen was made of $50 \times 40 \mathrm{~cm}$ barbed wire with $1 \times 1 \mathrm{~cm}$ areole. The trial started after the rats were placed on the horizontal screen on the ground. The screen was turned over $90^{\circ}$ within $2 \mathrm{sec}$ by raising one side of it gradually and maintaining this position for $5 \mathrm{sec}$. The time by which the rats took to hold onto the net screen was recorded in seconds. The scoring criterion was: 5 , holding on the screen and climbing upward; 4, holding on the screen with forelimbs and not falling down within $5 \mathrm{sec}$; 3 , holding on the screen temporally and slipping off a certain distance; 2 , falling down to the ground within $5 \mathrm{sec}$; 1 , falling down to the ground immediately as soon as the screen was set at a vertical position.

Measurement of infarct volumes. Following cerebral ischemia injury for seven days, the rats were anaesthetized with $10 \%$ chloral hydrate by intraperitoneal injection. The rats were perfused transcardiacally with $0.9 \% \mathrm{NaCl}$ and the brains were quickly removed for 2,3,5-triphenyl tetrazolium chloride (TTC) staining. Thereafter, six serial coronal sections of 2-mm thickness were prepared. Brain slices were incubated in a $0.2 \%$ TTC solution (Sigma, St. Louis, MO, USA) in phosphate-buffered saline (PBS) at $37^{\circ} \mathrm{C}$ for $20 \mathrm{~min}$ and fixed by immersion in $4 \%$ buffered formaldehyde solution. The normal area of brain was stained dark red based on intact mitochondrial function, whereas the infarct area remained unstained. Each brain slice was scanned by a high-resolution digital camera (Cannon Sx20), and the infarct volume was quantified using the Motic Med 6.0 System, which was represented as a percentage of the total brain volume.

$H$-reflex recording. The H-reflex was recorded as previously described (10). Briefly, the rats were anaesthetized with $10 \%$ chloral hydrate by intraperitoneal injection. The right hindlimbs of the rats were secured, and a pair of stimulating needle electrodes was transcutaneously inserted into the surroundings of the tibial nerve. Moreover, a pair of silver needle electrodes was inserted into the interosseous muscles between the fourth and the fifth or the first and the second metatarsal right foot muscles for recording. The tibial nerve was stimulated using square pulses with increasing stimulus intensity (0.1-10 in $0.5 \mathrm{~mA}$ increments, $0.1 \mathrm{~Hz}, 0.2 \mathrm{msec}$; RM6240; Chengdu Instrument Factory, Chengdu, China), and responses were recorded automatically. The threshold for both the $\mathrm{M}$ and $\mathrm{H}$ waves was determined, and the Hmax/Mmax ratio was calculated.

Determination of glutamate level in cerebrospinal fluid (CSF). The CSF was collected by cisternal puncture under light general anesthesia using $10 \%$ chloral hydrate by intraperitoneal injection. The derivatization process was performed by mixing $1 \mu 1$ of sample or glutamate standard solution, $1 \mu \mathrm{l}$ of freshly prepared methanolic OPA and $5 \mu \mathrm{l}$ borate buffer ( $\mathrm{pH} 9.5$ ). This final solution was vortexed and analyzed after $1 \mathrm{~min}$. The mobile phase that was used on the FLD system was composed of a mixture of $0.1 \mathrm{M}$ sodium acetate, $1.5 \mathrm{ml}$ tetrahydrofuran, $90 \mu 1$ triethylamine ( $\mathrm{pH} 7.20 \pm 0.05,1-2 \%$ acetic acid) and HPLC grade as phase A and methanol-acetonitrile-0.10.1 M sodium acetate $(\mathrm{pH} 7.20 \pm 0.05,1-2 \%$ acetic acid; $2: 2: 1 \mathrm{~V} / \mathrm{V})$ as phase B. Mobile phase and solvents were filtered through Millipore $0.45 \mu \mathrm{m}$ Durapore membrane filters and vacuum degassed prior to use. The gradient system was: $0 \mathrm{~min}, 0 \%$ mobile phase $\mathrm{B}$, increased to $60 \% \mathrm{~B}$ at $14 \mathrm{~min}(0.45 \mathrm{ml} / \mathrm{min})$, and held at $100 \% \mathrm{~B}$ until $15 \mathrm{~min}(0.8 \mathrm{ml} / \mathrm{min})$. The column was maintained at a temperature of $40^{\circ} \mathrm{C}$ and the fluorescence detector was set at $340 \mathrm{~nm}$ (excitation wavelength) and $450 \mathrm{~nm}$ (emission wavelength).

Western blot analysis. Ischemic cerebral tissues were homogenized in non-denaturing lysis buffer and centrifuged at $12,000 \mathrm{x} g$ for $15 \mathrm{~min}$. Supernatants were collected and frozen at $-80^{\circ} \mathrm{C}$ until immunoblot analysis. Protein concentration for each homogenate was determined. Equal amounts of protein $(50 \mu \mathrm{g})$ were loaded onto 8-10\% SDS-PAGE gels for electrophoresis and then transferred onto PVDF membranes. After blocking in $5 \%$ non-fat dry milk in $0.1 \mathrm{M}$ Tris-buffered saline (TBS)- $0.1 \%$ Tween-20 (TBST), the membranes were incubated with primary antibodies against GluR1, GluR2, GluR3, GluR4 and $\beta$-actin overnight on a shaker at $4^{\circ} \mathrm{C}$. Membranes were washed three times for $10 \mathrm{~min}$ with TBST and incubated for $1 \mathrm{~h}$ on a shaker at room temperature with goat anti-rabbit HRP-conjugated 


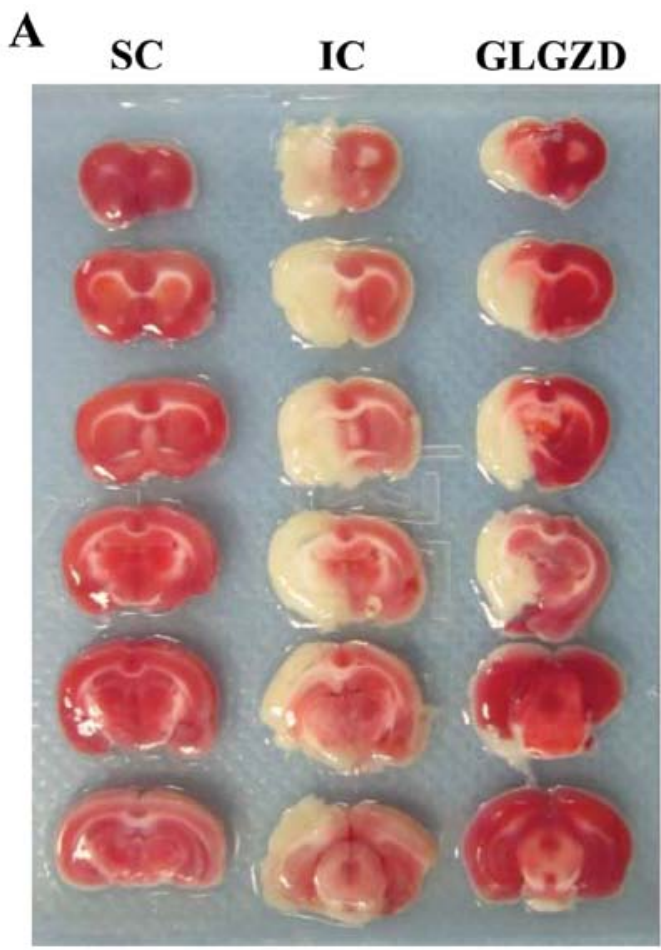

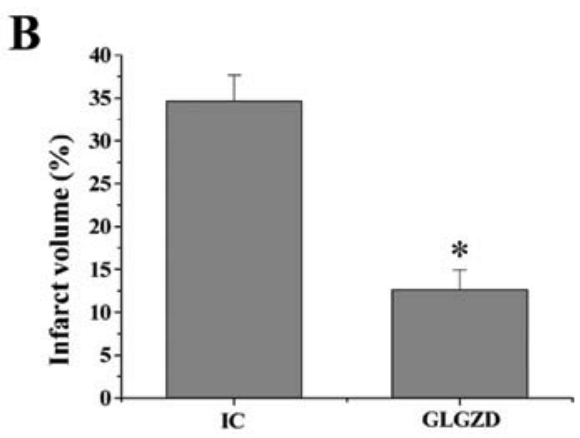

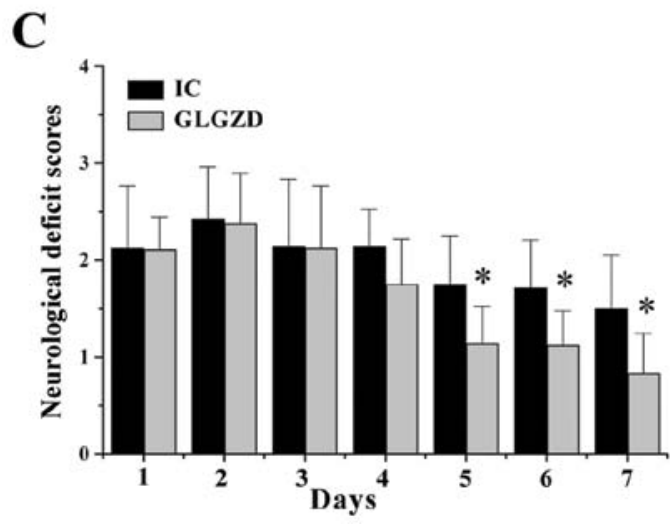

Figure 2. Effect of Gua Lou Gui Zhi decoction (GLGZD) on cerebral infarction and neurological deficits in cerebral ischemia/reperfusion (I/R)-injured rats. (A) At the end of the experiment, cerebral tissues from each group were coronally sectioned into 2 -mm-thick slices and then processed by TCC staining. The images were taken by a high-resolution digital camera. Images are representative of three independent experiments. SC, sham-operated control; IC, ischemic control. (B) Infarct volume was quantified using the Motic Med 6.0 System, which was represented as a percentage of the total brain volume. Data shown are the means \pm SE (error bars) from three individual rats in each group. ${ }^{*} \mathrm{P}<0.05$, vs. IC group. (C) Neurological deficits were examined daily in each group during the entire experiment. Data shown are the means $\pm \mathrm{SE}$ from eight individual rats in each group. ${ }^{*} \mathrm{P}<0.05$, vs. IC group.

secondary antibody. Blots were developed using enhanced chemiluminescence, and images were taken using a Bio-Image Analysis System (Bio-Rad, Hercules, CA, USA).

Perfusion fixation and fluorescent immunohistochemistry. The rats were anesthetized and perfused transcardiacally with $0.9 \%$ $\mathrm{NaCl}$ and $4 \%$ paraformaldehyde through the left ventricle and the brains were removed. Samples were fixed in cold $4 \%$ paraformaldehyde and then processed into $5-\mu \mathrm{m}$-thick sections. For staining, the slides were placed in PBS containing $10 \%$ normal goat serum (NGS), and incugated for $1 \mathrm{~h}$ at $37^{\circ} \mathrm{C}$ to block nonspecific protein activity. This was followed by an incubation at $4^{\circ} \mathrm{C}$ overnight with the primary antibodies, rabbit anti-GluR 1 (1:40), rabbit anti-GluR2 (1:50), rabbit anti-GluR3 (1: 300) and rabbit anti-GluR4 (1:300) (all from Abcam). After incubation with primary antibodies, the sections were washed three times in PBS and incubated with secondary goat anti-rabbit antibodies conjugated to a fluorescent marker (Alexa 488; Abcam). The nuclei of all cells were visualized by DAPI staining. After staining, the sections were dried at room temperature and covered with ProLong antifade medium (Invitrogen). Slides were analyzed using a Leica fluorescence microscope. Some slides were selected for confocal imaging using a confocal fluorescence microscope (Leiss LSM710).

Statistical analysis. Statistical data are expressed as the means \pm SD. Statistical analysis was performed using the Student's t-test and ANOVA using the SPSS package for
Windows (version 16.0). Differences with $\mathrm{P}<0.05$ were considered to be statistically significant.

\section{Results}

GLGZD ameliorates neurological deficits and cerebral infarction. Following I/R injury by $\mathrm{MCAO}$, the rats received GLGZD and its neuroprotective effects were evaluated by examining the neurological deficit scores. As expected, the rats in the SC group did not show any manifestation of neurological deficits (Fig. 2A), whereas all the rats in the IC and GLGZD-treated groups displayed obvious signs of cerebral injury ( $\mathrm{P}<0.05$, vs. SC group). However, after treatment with GLGZD for five days, the neurological deficit scores of the rats were significantly ameliorated (Fig. $2 \mathrm{~A})(\mathrm{P}<0.05$, vs. IC group). To further verify these results, we evaluated the effect of GLGZD on cerebral infarction. GLGZD treatment profoundly reduced cerebral infarct volumes in the I/R-injured rats ( $\mathrm{P}<0.05$, vs. IC group) (Fig. $2 \mathrm{~B}$ and $\mathrm{C}$ ). Taken together, these results deomonstrate that GLGZD exerts therapeutic effects against cerebral I/R injury.

GLGZD reduces cerebral ischemic spasticity. Cerebral I/R injury usually leads to spasticity, a motor disorder that is characterized by a velocity-dependent increase in muscle tone (hypertonia) exhibiting resistance to stretching. To evaluate the effect of GLGZD on cerebral ischemic spasticity, we performed a screen test to measure the muscle tone, strength, 


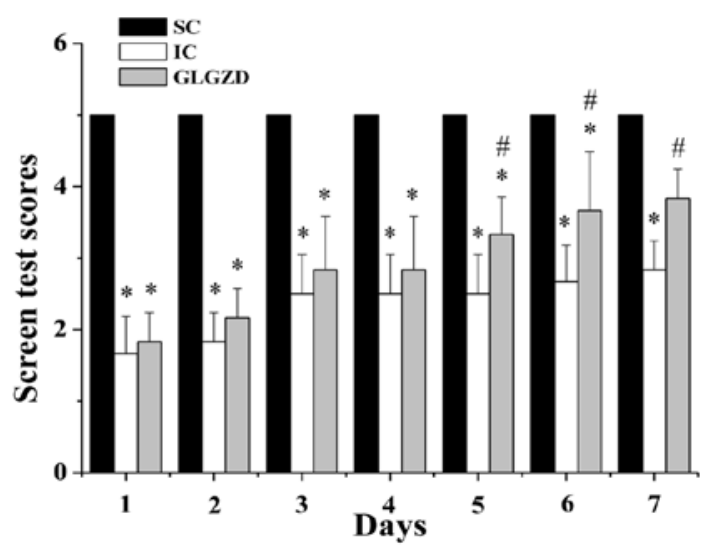

Figure 3. Effect of GLGZD on the screen test score in cerebral ischemia/ reperfusion I/R-injured rats. The screen test was carried out daily in each group during the entire study. Data shown are the means $\pm \mathrm{SE}$ from eight individual rats in each group. ${ }^{.} \mathrm{P}<0.05$, vs. $\mathrm{SC}$ group; ${ }^{*} \mathrm{P}<0.05$, vs. IC group.

stamina and balance in the rats from all the experimental groups. MCAO model construction significantly decreased the screen test score in the IC and GLGZD group rats $(\mathrm{P}<0.05$, vs. SC group), indicating that cerebral $\mathrm{I} / \mathrm{R}$ injury induced spasticity (Fig. 3). However, following treatment with GLGZD for five days, the screen test scores in the GLGZD-treated rats were significantly enhanced, as compared with those in the rats from the IC group $(\mathrm{P}<0.05)$, suggesting that GLGZD treatment alleviates the severity of cerebral ischemic spasticity.

To confirm the above observations, we determined the effect of GLGZD on the H-reflex that represents monosynaptic reflex and thus indicates the degree of excitability of motor neurons. Cerebral I/R injury resulted in an obvious increase in the amplitude and decrease in the latency of the H-reflex wave, as well as a significant increase in the Hmax/Mmax ratio, demonstrating the occurrence of spasticity (Fig. 4). However, GLGZD treatment ameliorated the spasticity in cerebral I/R-injured rats.

GLGZD inhibits $I / R$-induced elevation of glutamate levels in $C S F$. Glutamate is the principal fast excitatory neurotransmitter in the mammalian brain. Cerebral ischemia or brain injury has been shown to cause a marked elevation in glutamate concentrations (21), which is responsible for neuronal injury or death due to excitotoxicity (22). The suppression of excitation (suppression of glutamate levels) is considered as a

A

Day 1

SC

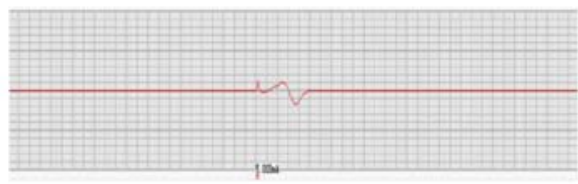

IC
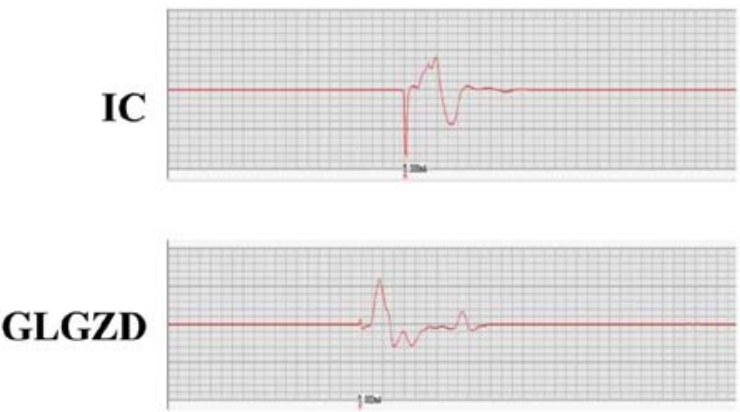

B

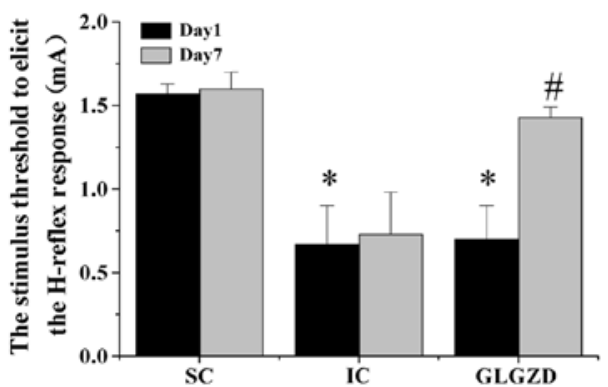

Day 7
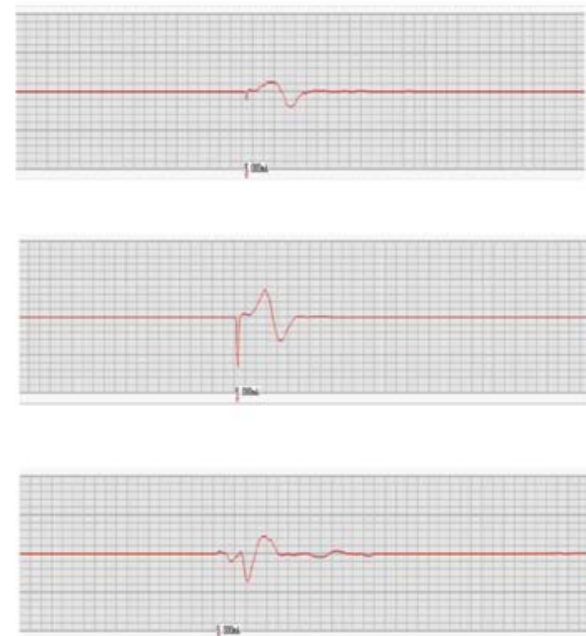

C

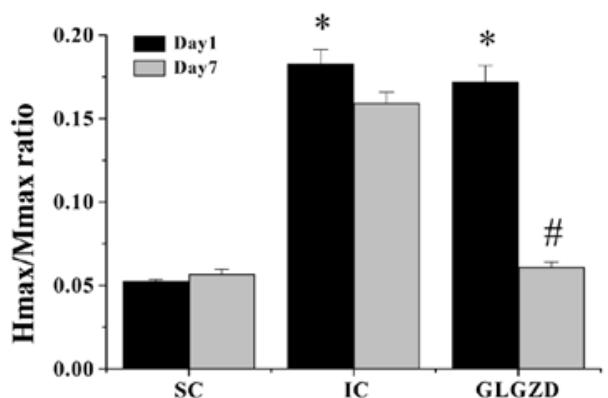

Figure 4. Effect of Gua Lou Gui Zhi decoction (GLGZD) on the H-reflex in cerebral ischemia/reperfusion (I/R)-injured rats. (A) Under the same stimulation of $5 \mathrm{~mA}$, the amplitude changes in the H-reflex waves between each group on days one and seven are shown. (B) The stimulus threshold to elicit the H-reflex response in each group, ${ }^{*} \mathrm{P}<0.05$, vs. SC group; ${ }^{\#} \mathrm{P}<0.05$, vs. IC group. (C) The Hmax/Mmax ratio between each group, ${ }^{*} \mathrm{P}<0.05$, vs. SC group; ${ }^{\#} \mathrm{P}<0.05$, vs. IC group. SC, sham-operated control; IC, ischemic control. 


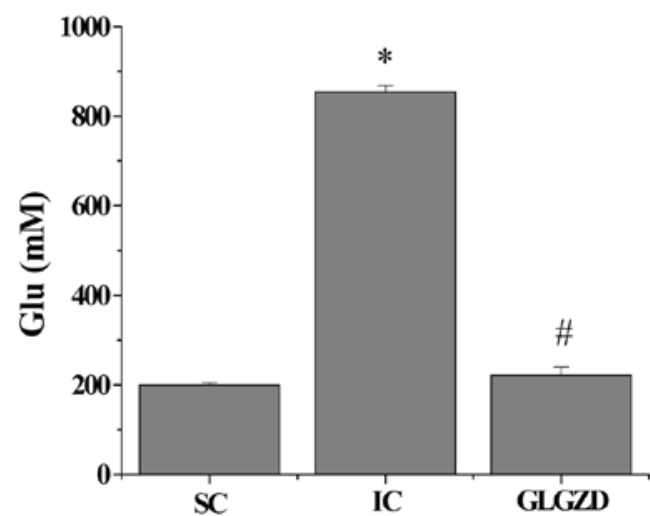

Figure 5. Effect of Gua Lou Gui Zhi decoction (GLGZD) on glutamate levels in cerebrospinal fluid (CSF) in cerebral ischemia/reperfusion (I/R)-injured rats. The CSF levels of glutamate were examined by HPLC. Data are the means \pm SE (error bars) of five individual rats in each group. ${ }^{*} \mathrm{P}<0.05$, vs. $\mathrm{SC}$ group; ${ }^{\mathrm{P}} \mathrm{P}<0.05$, vs. IC group. SC, sham-operated control; IC, ischemic control.

means of treating spasticity $(7,8)$. By HPLC analysis, we found that cerebral I/R injury significantly enhanced the glutamate concentration in the CSF (Fig. 5) (SC group, 201.16 $\pm 3.69 \mathrm{mM}$; IC group, $855.05 \pm 13.20 \mathrm{mM} ; \mathrm{P}<0.05)$. However, the administration of GLGZD markedly reduced the levels of glutamate to $222.97 \pm 16.93 \mathrm{mM}(\mathrm{P}<0.05$, vs. IC group $)$.

GLGZD alters the expression of AMPA receptor subunits following cerebral ischemia. AMPA receptors are one of the ionotropic glutamate receptors for the fast excitatory synaptic transmission in the CNS. AMPA subunit expression is altered in cerebral ischemia tissues (9), playing an important role in ischemic spasticity (10). To further explore the mechanisms mediating the neuroprotective and anti-spasticity effects of GLGZD, we examined its effect on the expression of AMPA receptor subunits in ischemic cerebral tissues. Data from both western blot analysis and immunofluorescence showed that, as compared with the SC group, the protein expression of GluR1, GluR3 and GluR4 was increased, whereas that of GluR2 was reduced in the rats from the IC group. However, the I/R-induced alteration in the expression of AMPA receptor subunits was neutralized by GLGZD treatment (Figs. 6 and 7).

\section{Discussion}

Spasticity is a serious post-stroke physical disability and may slow down the potential success of rehabilitation. Glutamate and AMPA receptors have been shown to play a crucial role in spasticity following cerebral I/R injury. Glutamate is one of the most abundant excitatory neurotransmitters in the mammalian CNS and is responsible for sending signals between nerve cells. At normal concentrations it plays a critical role in learning and memory. However, under pathological conditions such as ischemic stroke, glutamate in the extracellular fluid usually accumulates to reach aberrantly high concentrations, which can lead to overexcitation and eventually the death of nerve cells. This pathological process is termed excitotoxicity that is associated with spasticity $(7,23)$. As well as pathologically high glutamate levels, excitotoxicity can be induced by the overactivation of glutamate receptors, such as the AMPA receptors. The

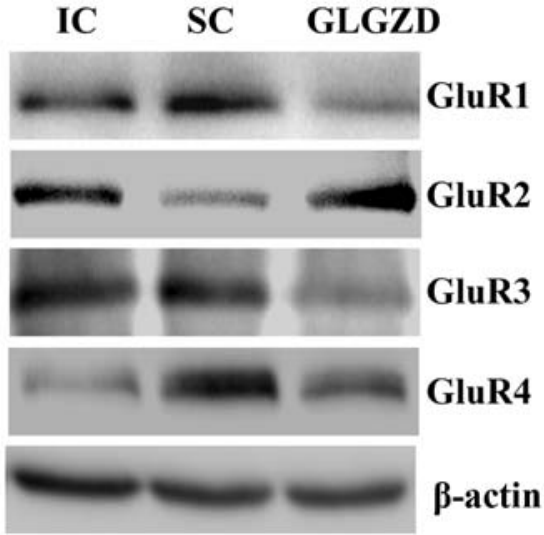

Figure 6. Effect of Gua Lou Gui Zhi decoction (GLGZD) on the expression of the AMPA receptor subunits (western blot analysis). Plotein expression levels of AMPA receptor subunits in ischemic cerebral tissues were determined by western blot analysis. $\beta$-actin was used as the internal control. Data are representative of five individual rats in each group.

binding of glutamate to its receptors can cause high levels of $\mathrm{Ca}^{2+}$ to influx into cells, initiating the process of cell apoptosis. AMPA receptors are composed of four types of subunits (known as GluR1-4) that mostly exist as heterotetramers, consisting of symmetric 'dimer of dimers' of GluR2 and either GluR1, GluR3 or GluR4. Although the four subunits of AMPA receptor family are of similar size and are approximately $70 \%$ homologous, their functions differ. Unlike the GluR1, GluR3 and GluR4 subunits that facilitate $\mathrm{Ca}^{2+}$ influx, the GluR2 subunit almost always prevents calcium from entering the cell. Therefore, the permeability of AMPA receptors to $\mathrm{Ca}^{2+}$ is determined by the GluR2 subunit. If an AMPA receptor lacks a GluR2 subunit, it will be permeable to $\mathrm{Ca}^{2+}$; whereas GluR2-containing AMPA receptors are unfavorable for calcium influx. AMPA receptor subunits have been found on spinal $\alpha$-motoneurons as well as on presynaptic Ia afferents, consistent with their demonstrated role in motor function. It has been shown that the expression of AMPA receptors can affect the clinical signs of spasticity and rigidity following cerebral ischemia; the intrathecal or systemic delivery of the selective AMPA receptor antagonist, NGX424, represents an effective therapy for modulating chronic spasticity in baclofen-tolerant animals $(24,25)$.

GLGZD is a classical TCM that was first prescribed in the Eastern Han Dynasty, around 210 AD. As shown in our previous study (unpublished data), GLGZD exerts significant therapeutic effects on spasticity in stroke patients. However, the mode of action of its neuroprotective and anti-spasticity effects remains poorly understood. In the present study, using a focal cerebral ischemia rat model, we demonstrate that GLGZD exerts neuroprotective effects by improving neurological deficits and reducing the cerebral infarct volume. In addition, GLGZD displays anti-spasticity effects by improving the screen test and H-reflex scores. Moreover, our results demonstrate that GLGZD significantly decreases the cerebral I/R-induced overexpression of glutamate in CSF. Furthermore, GLGZD downregulates the expression of the AMPA receptor subunits, GluR1, GluR3 and GluR4, but increases GluR2 expression in cerebral I/R-injured rats.

In conclusion, to our knowledge, in the present study, we report for the first time that GLGZD exerts neuroprotective 

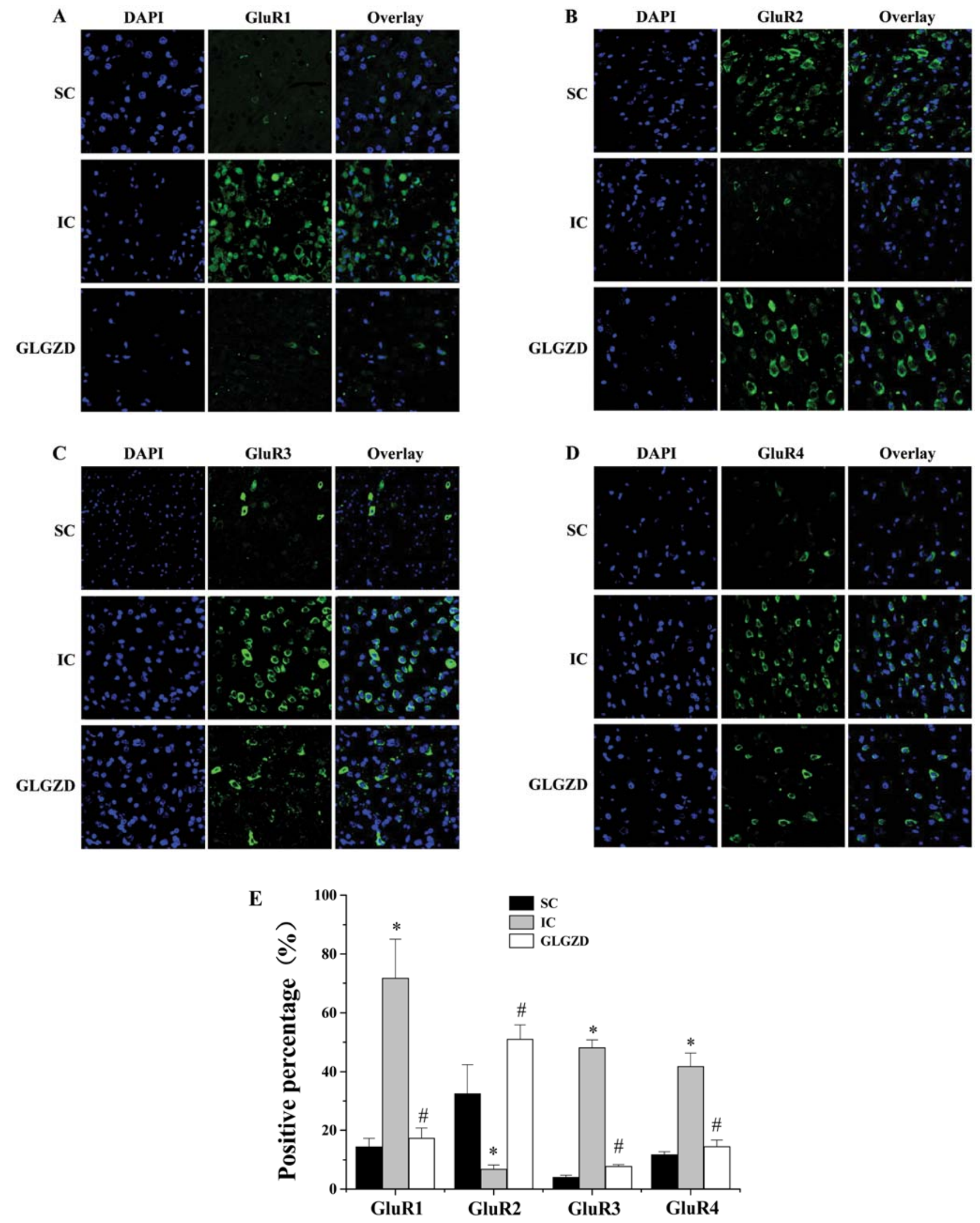

Figure 7. Effect of Gua Lou Gui Zhi decoction (GLGZD) on AMPA expression using fluorescent immunohistochemistry. At the end of the experiment, cerebral tissues from each group $(n=5)$ were processed for fluorescent immunohistochemistry. Nuclei of all cells were visualized by DAPI staining and the green fluorescence of AMPA receptors was detected by a confocal fluorescence microscope. AMPA-positive cells were counted at four arbitrarily selected microscopic fields at a magnification of x400. SC, sham operation control; IC, ischemic control; GLGZD, Gua Lou Gui Zhi Decoction. (A) Fluorescent microscope images showing GluR1-immunoreactive cells (green) and nucleus of neurons (blue) between different groups. After ischemia, strong expression of GluR1 can be seen while after treatment of GLGZD, expression of GluR1 was decreased. (B) GluR2-immunoreactive cells (green) and nucleus of neurons (blue) of different groups. After ischemia, decreased expression of GluR2 was seen. After GLGZD, expression of GluR2 was increased. (C) Strong GluR3-immunoreactive cells was evident after ischemia. After GLGZD, expression of GluR3 was decreased. (D) GluR4-immunoreactive cells was also noted to be strongly expressed after ischemia. After GLGZD, expression of GluR4 was decreased. (E) Positive rate was expressed as the ratio of green-stained cells to the blue DAPI-stained total cells. Data are the means $\pm \mathrm{SE}$ (error bars). ${ }^{*} \mathrm{P}<0.05$, vs. SC group; ${ }^{*} \mathrm{P}<0.05$, vs. IC group. 
and therapeutic effects against spasticity in an ischemic stroke model via the inhibition of glutamate/AMPA receptor-mediated excitotoxicity. These data suggest that GLGZD may be a potential therapeutic agent for cerebral ischemia and spasticity.

\section{Acknowledgements}

This study was sponsored by the Guidance Project of the Fujian Provincial Department of Science and Technology (no. 2012D011), and the Key Project of the Department of Health of Fujian Province (no. zlckf01).

\section{References}

1. Mayer NH, Esquenazi A and Childers MK: Common patterns of clinical motor dysfunction. Muscle Nerve Suppl 20: S21-S35, 1997.

2. Lance JW: What is spasticity? Lancet 335: 606, 1990.

3. Lundstrom E, Terent A and Borg J: Prevalence of disabling spasticity 1 year after first-ever stroke. Eur J Neurol 15: 533-539, 2008.

4. Watkins CL, Leathley MJ, Gregson JM, Moore AP, Smith TL and Sharma AK: Prevalence of spasticity post stroke. Clin Rehabil 16: 515-522, 2002.

5. Sommerfeld DK, Eek EU, Svensson AK, Holmqvist LW and von Arbin MH: Spasticity after stroke: its occurrence and association with motor impairments and activity limitations. Stroke 35: 134-139, 2004.

6. Duncan PW, Zorowitz R, Bates B, et al: Management of adult stroke rehabilitation care a clinical practice guideline. Stroke 36 e100-e143, 2005

7. Gracies JM, Nance P, Elovic E, McGuire J and Simpson DM Traditional pharmacological treatments for spasticity. Part II: General and regional treatments. Muscle Nerve Suppl 20: S92-S120, 1998

8. Davidoff RA: Antispasticity drugs: mechanisms of action. Ann Neurol 17: 107-116, 2004

9. Gottlieb M and Matute C: Expression of ionotropic glutamate receptor subunits in glial cells of the hippocampal CA1 area following transient forebrain ischemia. J Cereb Blood Flow Metab 17: 290-300, 1997.

10. Hefferan MP, Kucharova K, Kinjo K, et al: Spinal astrocyte glutamate receptor 1 overexpression after ischemic insult facilitates behavioral signs of spasticity and rigidity. J Neurosci 27 : 11179-11191, 2007.
11. Saulino M and Jacobs BW: The pharmacological management of spasticity. J Neurosci Nurs 38: 456-459, 2006.

12. Hesse S and Werner C: Poststroke motor dysfunction and spasticity: novel pharmacological and physical treatment strategies. CNS Drugs 17: 1093-1107, 2003.

13. Meythaler JM, Guin-Renfroe S, Johnson A and Brunner RM Prospective assessment of tizanidine for spasticity due to acquired brain injury. Arch Phys Med Rehabil 82: 1155-1163, 2001.

14. Meythaler JM, Guin-Renfroe S, Law C, Grabb P and Hadley MN: Continuously infused intrathecal baclofen over 12 months for spastic hypertonia in adolescents and adults with cerebral palsy. Arch Phys Med Rehabil 82: 155-161, 2001.

15. Ford B, Greene P, Louis ED, et al: Use of intrathecal baclofen in the treatment of patients with dystonia. Arch Neurol 53: 1241-1246, 1996.

16. Sun X: Research on formula treating paralysis and spasticity from 'treatise on febrile and miscellaneous diseases'. Zhongguo Zhong Yi Ji Chu Yi Xue Za Zhi 8: 644-645, 2010 (In Chinese).

17. Zhang L and Ai H: Effects of Gua Lou Gui Zhi decoction on c-fos and c-jun in epileptic rats. Sichuan Hua xi Zhong Yi Yao Yan Jiu Suo 23: 21-22, 2005 (In Chinese).

18. Yang C, Chen L and Tao J: New usage of a classical formula - Gua Lou Gui Zhi decoction. Liaoning Zhong Yi Za Zhi 8: 166-167, 2012 (In Chinese).

19. Longa EZ, Weinstein PR, Carlson S and Cummins R: Reversible middle cerebral artery occlusion without craniectomy in rats. Stroke 20: 84-91, 1989.

20. Guo J, Liu L, Ma C, Xu B, Duan X and Wang B: Effect of restraint stress on depression-like behaviors in rats after transient focal cerebral ischemic injury. Neural Regen Res 2: 390-394, 2007.

21. Parelkar NK and Wang JQ: Upregulation of metabotropic glutamate receptor $8 \mathrm{mRNA}$ expression in the rat forebrain after repeated amphetamine administration. Neurosci Lett 433: 250-254, 2008.

22. Bonde C, Noraberg J, Noer H and Zimmer J: Ionotropic glutamate receptors and glutamate transporters are involved in necrotic neuronal cell death induced by oxygen-glucose deprivation of hippocampal slice cultures. Neuroscience 136: 779-794, 2005.

23. Abbruzzese G: The medical management of spasticity. Eur J Neurol 9 (Suppl 1): S30-S34, 2002.

24. Oshiro M, Hefferan MP, Kakinohana O, et al: Suppression of stretch reflex activity after spinal or systemic treatment with AMPA receptor antagonist NGX424 in rats with developed baclofen tolerance. Br J Pharmacol 161: 976-985, 2010.

25. Gómez-Soriano J, Goiriena E and Taylor J: Spasticity therapy reacts to astrocyte GluA1 receptor upregulation following spinal cord injury. Br J Pharmacol 161: 972-975, 2010. 